\title{
Low skeletal muscle mass is associated with mortality in kidney transplant recipients
}

\author{
Juhan Lee ${ }^{1}$, Hyun Jeong Kim¹, Beom Seok Kim², Myoung Soo Kim¹, Soon II Kim¹, Kyu Ha Huh \\ ${ }^{1}$ Department of Surgery-Transplantation, Yonsei University College of Medicine, Seoul, Korea \\ ${ }^{2}$ Department of Internal Medicine-Nephrology, Yonsei University College of Medicine, Seoul, Korea
}

Background: Muscle wasting in chronic kidney disease is associated with increased cardiovascular events, morbidity, and mortality. However, whether pre-transplant skeletal muscle mass affects kidney transplant outcomes remains undetermined.

Methods: To examine the impact of skeletal muscle mass on transplant outcomes, we analyzed 623 patients who underwent kidney transplantation between 2004 and 2019. We measured the cross-sectional area of total skeletal muscle at the third lumbar region from a pre-transplant computed tomography scan. Low muscle mass was defined as the sex-specific lowest quartile of the skeletal muscle index.

Results: During the follow-up period, 44 patients $(7.1 \%)$ died and 54 patients $(8.7 \%)$ experienced death-censored graft loss. The 1-year, 3 -year, and 5-year overall graft survival rates were $92.9 \%, 87.4 \%$, and $82.8 \%$ for the low muscle mass group and $96.6 \%$, $93.8 \%$, and $91.4 \%$ for the high muscle mass groups, respectively $(P=0.003)$. A multivariable Cox regression analysis confirmed that low muscle mass was independently associated with all-cause mortality (hazard ratio [HR], 3.881; 95\% confidence interval [Cl], 1.613-9.336; $\mathrm{P}=0.002)$ and overall graft loss $(\mathrm{HR}, 2.329 ; 95 \% \mathrm{Cl}, 1.301-4.169 ; \mathrm{P}=0.004)$. By contrast, death-censored graft survival rates were comparable between low and high muscle mass groups. Low muscle mass was also associated with an increased risk of hospital readmission within 1 year after transplant.

Conclusions: Pre-transplant low skeletal muscle mass is associated with increased risk mortality and hospital readmission after kidney transplantation.

Corresponding author: Juhan Lee

E-mail: laplaine@yuhs.ac

\footnotetext{
(C) The Korean Society for Transplantation

This is an Open Access article distributed under the terms of the Creative Commons Attribution Non-Commercial License (http://creativecommons.org/licenses/by-nc/4.0/) which permits unrestricted non-commercial use, distribution, and reproduction in any medium, provided the original work is properly cited.
} 